Article

\title{
Aerodynamic Performance of Hex-Rotor UAV Considering the Horizontal Airflow
}

\author{
Yao Lei ${ }^{1,2, *(D)}$ and Mingxin Cheng ${ }^{1}$ \\ 1 School of Mechanical Engineering and Automation, Fuzhou University, Fuzhou 350116, China; \\ mingxincheng2019@gmail.com \\ 2 Key Laboratory of Fluid Power and Intelligent Electro-Hydraulic Control (Fuzhou University), \\ Fujian Province University, Fuzhou 350116, China \\ * Correspondence: yaolei@fzu.edu.cn
}

Received: 12 October 2019; Accepted: 6 November 2019; Published: 9 November 2019

Featured Application: This paper tackles an aerodynamic problem of Hex-rotors UAV considering the wind effect and aerodynamic interference which is applicable to a wider class in progress to verify the effectiveness of flight control model, and the applicability of the concept to control in forward flight and under wind disturbances will be proven in our further studies.

\begin{abstract}
In this paper, the aerodynamic performance of a Hex-rotor unmanned aerial vehicle (UAV) with different rotational speeds (1500-2300 RPM) considering the horizontal airflow conditions is analyzed by both simulations and experiments. A low-speed wind tunnel experiments platform is applied to measure the thrust, torque, and power consumption of a Hex-rotor UAV with different rotational speeds in horizontal airflow, which varied from $0 \mathrm{~m} / \mathrm{s}-4 \mathrm{~m} / \mathrm{s}$. First, this paper introduces the effect of horizontal airflow on a UAV. Then, the low-speed wind tunnel experiments were carried out on a Hex-rotor UAV ( $\mathrm{D} / \mathrm{L}=0.56)$ with different horizontal velocities to determine the hover performance. Finally, numerical simulations were obtained with the streamline distributions, pressure distributions, velocity contour, and vortex distributions at different horizontal airflow conditions to describe the aerodynamic interference effect of different horizontal airflows. Combined with the experimental results and numerical simulations results, the horizontal airflow proved to have a significant influence on the aerodynamic performance of the Hex-rotor UAV with an increase in thrust and power. Indeed, the streamlines in the flow field were coupled to each other at the presence of the incoming airflow. Especially when the incoming airflow was larger, the Hex-rotor UAV could properly use low-speed flight to maintain high power loading. Finally, it is inferred that the aerodynamic performance of the Hex-rotor $\mathrm{UAV}$ is also related to the movement and deformation of the vortex at the tip of the rotor.
\end{abstract}

Keywords: Hex-rotor UAV; horizontal airflow; numerical simulation; low-speed wind tunnel

\section{Introduction}

A Hex-rotor unmanned aerial vehicle (UAV) is designed with the advantages of a simple structure, a stable system, and strong flight adaptability, which has wide civil and military applications [1-6]. Considering the manipulation of the Hex-rotor UAV, the horizontal movement can only change the rotational speed of the different rotors of the Hex-rotor UAV through a controller, thereby completing tasks such as acceleration and hovering. Also, the external airflow disturbance and the mutual interference between the rotors affects the aerodynamic performance of the Hex-rotor UAV. The vortices shed the rotor of the Hex-rotor UAV and form a wide range of mutual interference areas under the $\mathrm{UAV}$, and the aerodynamic performance of the Hex-rotor UAV will be affected. Therefore, it is very 
important to the Hex-rotor UAV to analyze the aerodynamic characteristics in the horizontal airflow. In addition, when the horizontal airflow is introduced, the incoming airflow will interfere with the aerodynamic characteristics between the adjacent rotors, making the flow field of the Hex-rotor UAV more complicated, affecting the stability and reliability of the Hex-rotor UAV.

However, most studies on the Hex-rotor UAV are aimed at attitude control and path planning. Pflimlin et al. describes a control strategy on UAV vertical takeoff and landing (VTOL) [7]. Ma et al. studied a nonlinear dynamic model of a miniature Hex-rotor UAV using MATLAB simulation (Matlab2012, MathWorks, Natick, MA, 2012) as the control algorithm [8]. Lee et al. studied the aerial manipulation system of the Hex-rotor UAV [9]. Zhuo et al. performed attitude control on the Hex-rotor unmanned aerial vehicle (UAV) [10]. Shi et al. studied Hex-rotor UAV indoor flight path planning [11]. Kirsch et al. studied the nonlinear control of a Hex-rotor UAV [12]. Zhao et al. researched a control system designed using neural network algorithms [13].

Currently, only a few researchers pay attention to the aerodynamic characteristics of a Multi-rotor UAV in the incoming airflow conditions. Zheng et al. studied the general characteristics of the distribution of the downwash airflow and the rotation flow fields around the rotor tip [14]. Hrishikeshavan studied flight performance tests of MAVs under wing [15]. Chang-Jun et al. applied the controller to obtain the height error and attitude error in the environment of airflow disturbance and verified the effectiveness through aerodynamic analysis [16]. Lei et al. studied the aerodynamic performance of a non-planar Quadrotor UAV [17]. These results are all introduced to understand the aerodynamic characteristics of the Hex-rotor UAV. However, the aerodynamics of a Hex-rotor UAV under external airflow and rotor interference have not yet been studied. Above all, it is necessary to study the aerodynamic characteristics of the Hex-rotor UAV considering the horizontal wind effect.

This paper is structured as follows: the aerodynamic analysis of the Hex-rotor UAV is presented in Section 2. In Section 3, experiments with different horizontal airflow through a custom-designed experiments platform are introduced in detail. In Section 4, numerical simulations of aerodynamics of a Hex-rotor UAV are performed. Finally, Section 5 gives the conclusions.

\section{Theoretical Analysis}

In the natural environment, light breeze $(1.6-3.3 \mathrm{~m} / \mathrm{s})$ and gentle breeze $(3.4-5.4 \mathrm{~m} / \mathrm{s})$ are the most common wind speeds in the urban city. The common wind speed does not exceed $5 \mathrm{~m} / \mathrm{s}$, so the average value of the light breeze at $2.5 \mathrm{~m} / \mathrm{s}$ and the gentle breeze at $4 \mathrm{~m} / \mathrm{s}$ are introduced as the incoming wind speed to study the effect of horizontal airflow interference $[18,19]$. In addition, the case of no horizontal airflow $(0 \mathrm{~m} / \mathrm{s})$ is also selected as the comparison.

In this study, the power loading $(P L)$ is applied to evaluate the hovering performance of the Hex-rotor UAV, which is defined as follows [20]:

$$
P L=\frac{C_{T}^{\frac{3}{2}}}{C_{P} \sqrt{2}}=\frac{T^{\frac{3}{2}}}{P \sqrt{2 \rho A}}
$$

where the thrust coefficient is defined as:

$$
C_{T}=\frac{T}{\rho A \Omega^{2} R^{2}}
$$

and the power coefficient is:

$$
C_{P}=\frac{P}{\rho A \Omega^{3} R^{3}}=\frac{Q \Omega}{\rho A \Omega^{3} R^{3}}=\frac{Q}{\rho A \Omega^{2} R^{3}}
$$

where $\rho, A, T, P$ and $Q$ are the air density $\left(\mathrm{kg} / \mathrm{m}^{3}\right)$, rotor area $\left(\mathrm{m}^{2}\right)$, thrust $(\mathrm{N})$, power $(\mathrm{W})$, and torque $(\mathrm{Nm})$, respectively. 
Therefore, $P L$ can be simplified as:

$$
P L=\frac{C_{T}^{\frac{3}{2}}}{C_{P} \sqrt{2}}=\frac{T^{\frac{3}{2}}}{P \sqrt{2 \rho A}}
$$

It is easily shown that the higher hover efficiency with better aerodynamics is described by a higher $P L$, which means a larger thrust and a smaller power consumption.

\section{Experiments}

\subsection{Basic Parameters}

The propeller is made of carbon fiber with a diameter of $400 \mathrm{~mm}$ and a weight of about $0.015 \mathrm{~kg}$. The propeller has two blades and the rotation speed ranged from 1500 RPM to 2300 RPM. Therefore, the blade tip Mach number is $0.1-0.14$ and the blade tip Reynolds number is $0.8 \times 10^{5}-1.2 \times 10^{5}$. The diagram of the propeller is shown in Figure 1. The characteristics of the propeller are shown in Table 1.

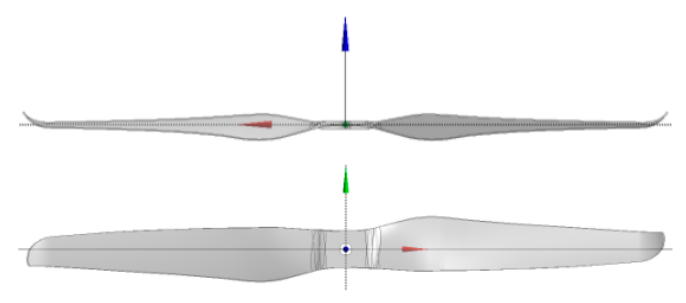

Figure 1. Propeller profile.

Table 1. The characteristics of the propeller. RPM: Revolutions Per Minute

\begin{tabular}{ccccccc}
\hline Radius (m) & Pitch (m) & Twist Angle & RPM & $\begin{array}{c}\text { Mach } \\
\text { Number }\end{array}$ & $\begin{array}{c}\text { Reynolds } \\
\text { Number (10 })^{5}\end{array}$ & $\begin{array}{c}\text { Max } \\
\text { Camber }\end{array}$ \\
\hline 0.2 & 0.157 & 0 & $1500-2400$ & $0.1-0.14$ & $0.8-1.2$ & $5.5 \%$ \\
\hline
\end{tabular}

\subsection{Experimental Setup}

According to the theoretical analysis, we need to measure the Revolutions Per Minute (RPM), thrust, torque, and power consumption (current and voltage) to obtain the PL during the experiments. In addition, a low-speed wind tunnel was applied to simulate the horizontal airflow, and the wind speed was set as $0 \mathrm{~m} / \mathrm{s}, 2.5 \mathrm{~m} / \mathrm{s}$, and $4 \mathrm{~m} / \mathrm{s}$. The experimental setup is shown in Figure 2 .

As shown in Figure 2, the main measurement components include: (1) Optical tachometer (model: DT-2234C, accuracy: $6 \pm(0.05 \%+1 \mathrm{~d}))$ was applied to record the rotation speed; (2) thrust sensor (model: CZL605, accuracy: 0.02\% F.S) converted the thrust signal into an electrical signal output; (3) torque sensor (model: HLT-131, Accuracy: 0.5\% F.S) was fixed on the motor to obtain rotor torque as measured by resistance strain gauge technology. The signal process is shown in Figure 3. 


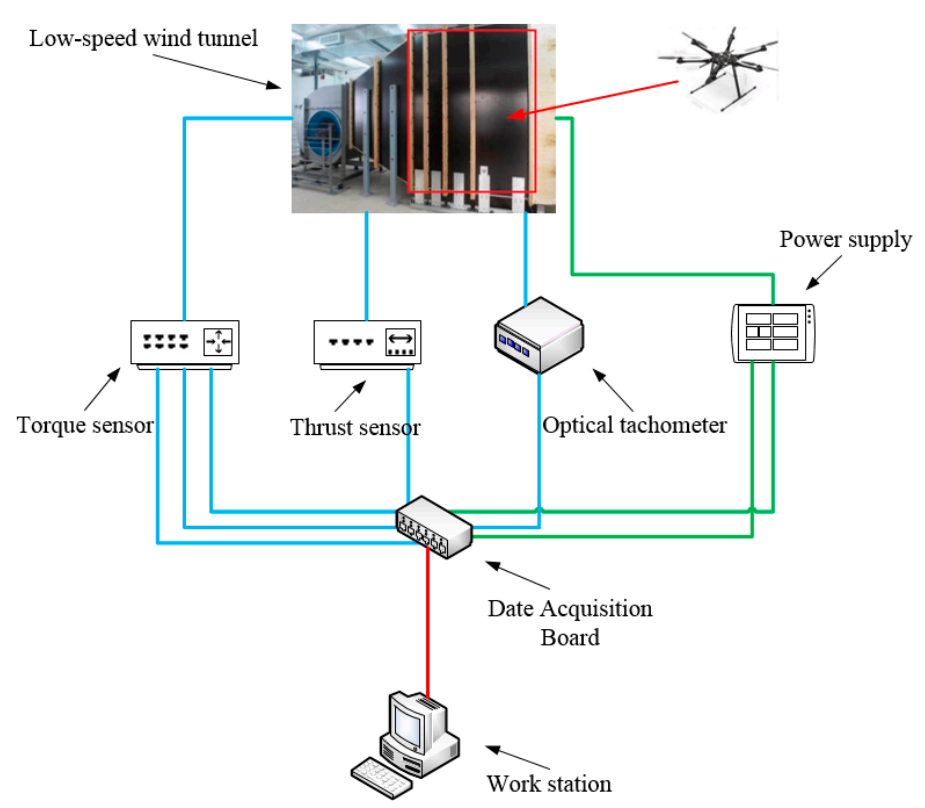

Figure 2. Experimental setup.

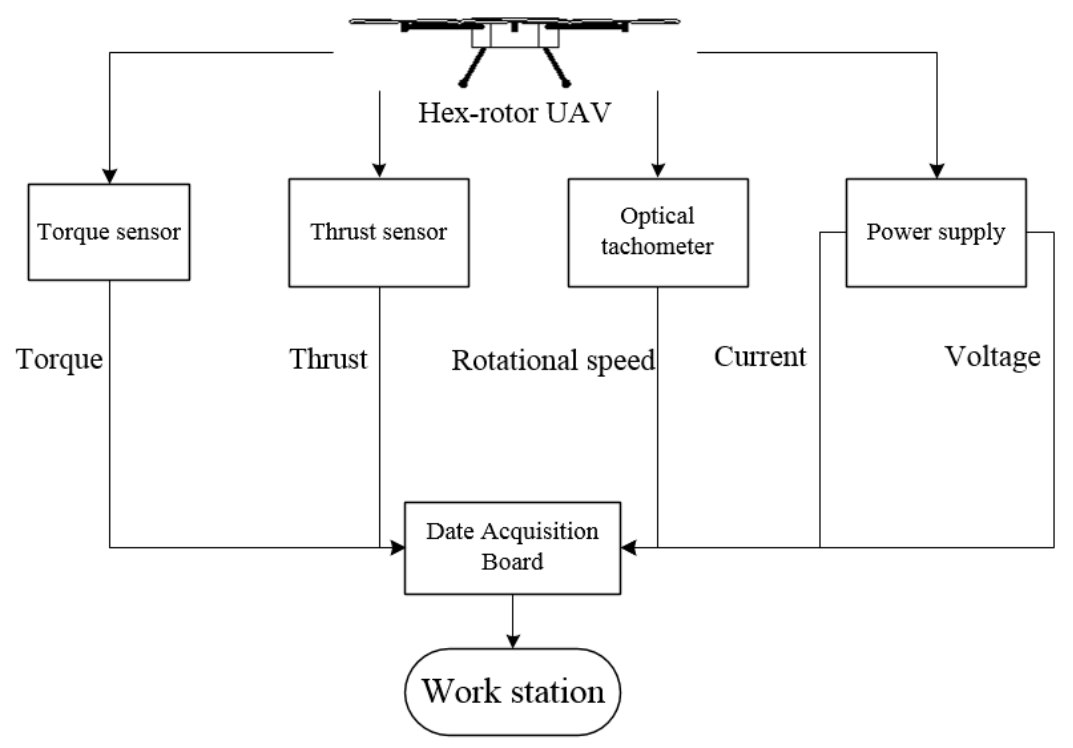

Figure 3. Sketch of the signal process.

\subsection{Experimental Results}

As the key parameters to analyze the hover efficiency of the Hex-rotor UAV, the thrust and power consumption are obtained with different wind speeds in Figure 4.

According to Figure 4, it is interesting to note that the thrust increased with wind speed, especially with a higher rotor speed. However, the power presented an opposite trend. This is because the interference between the rotors is much stronger when the horizontal airflow is introduced, which may improve the aerodynamic performance by increasing thrust and decreasing power.

Thrust and power increments of the Hex-rotor UAV in the low-speed wind tunnel experiments are shown in Figure 5. 


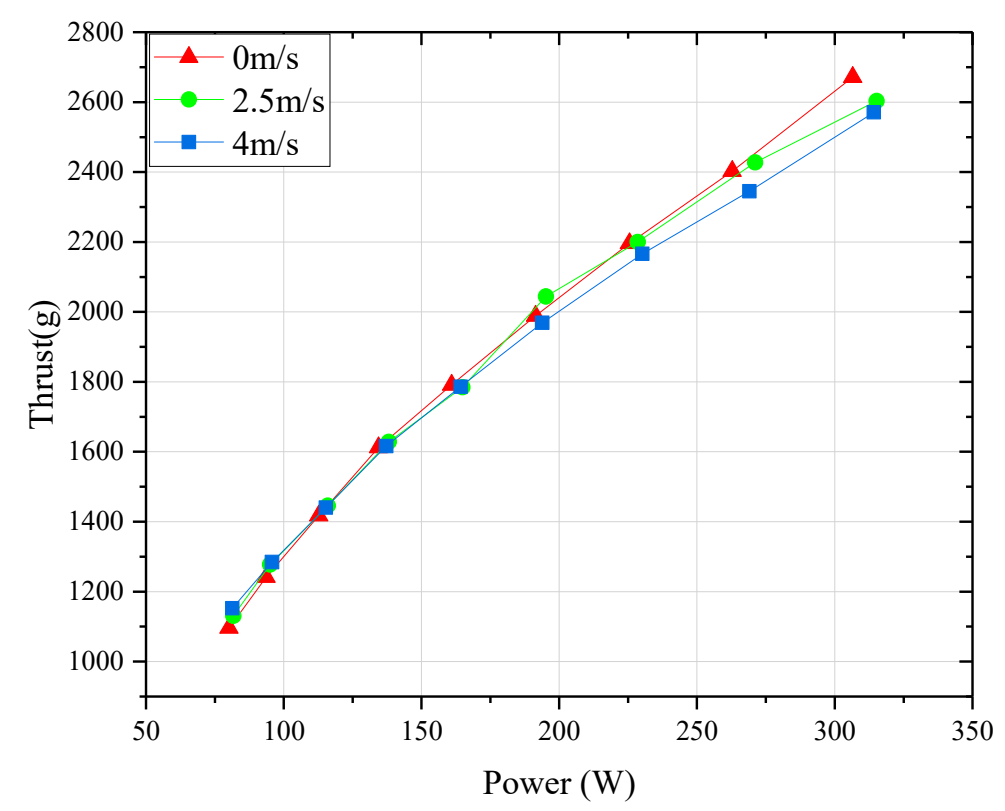

Figure 4. Thrust and power consumption with different wind speeds.

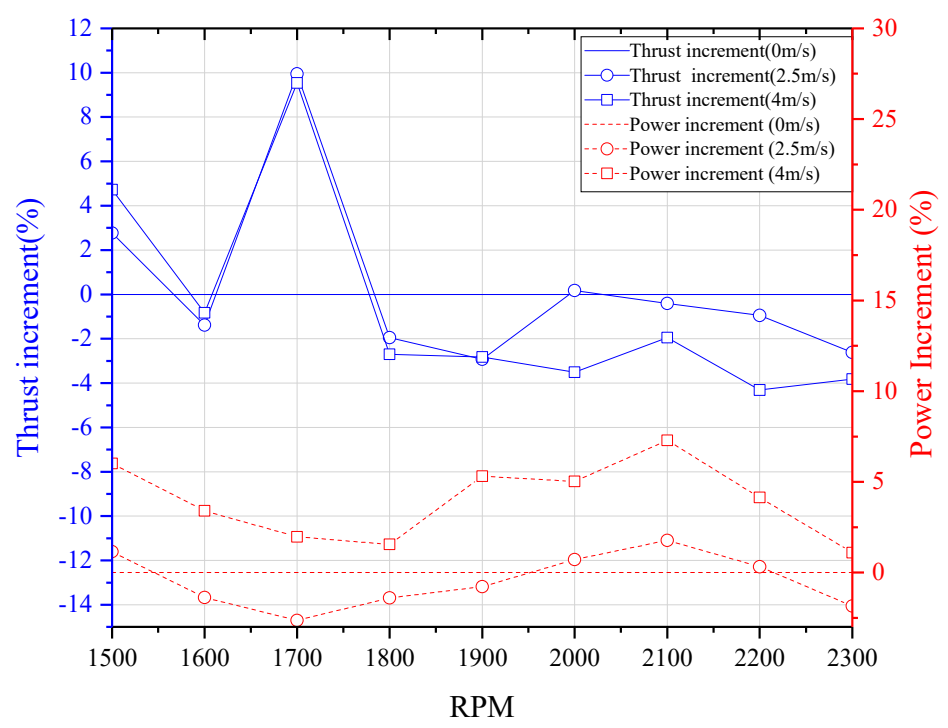

Figure 5. Thrust and power increment at different speeds.

As shown in Figure 5, the Hex-rotor UAV showed a good aerodynamic performance at a lower rotor speed arranged from 1600 to 1900 RPM. Especially at 1700 RPM, the UAV reached the best performance with the highest thrust increment and lowest power increment. As the rotor speed increased (1900 RPM-2300 RPM), the hover efficiency decreased with the wind speed since the thrust generated by the same speed gradually decreased, and the power consumption increased instead. Compared to thrust at $0 \mathrm{~m} / \mathrm{s}$, at the horizontal airflows of $2.5 \mathrm{~m} / \mathrm{s}$ and $4 \mathrm{~m} / \mathrm{s}$, the thrust decreased by a maximum of $2.92 \%$ and $3.82 \%$, respectively. Also, the thrust of the Hex-rotor UAV was reduced by $4.31 \%$ and the power consumption was increased by $4.13 \%$ when the horizontal airflow was $4 \mathrm{~m} / \mathrm{s}$ at 2200 RPM (work mode). The main reasons are: (1) at a lower rotor speed, the interference between the rotors domain the whole aerodynamic environment where the wind effect was relatively small at this stage, (2) at a higher rotor speed, the downwash began to couple with the incoming flow. In this case, the shape of the downwash deformed which may have increased the power consumption.

Power loading at different horizontal airflows and RPM is shown in Figure 6. 


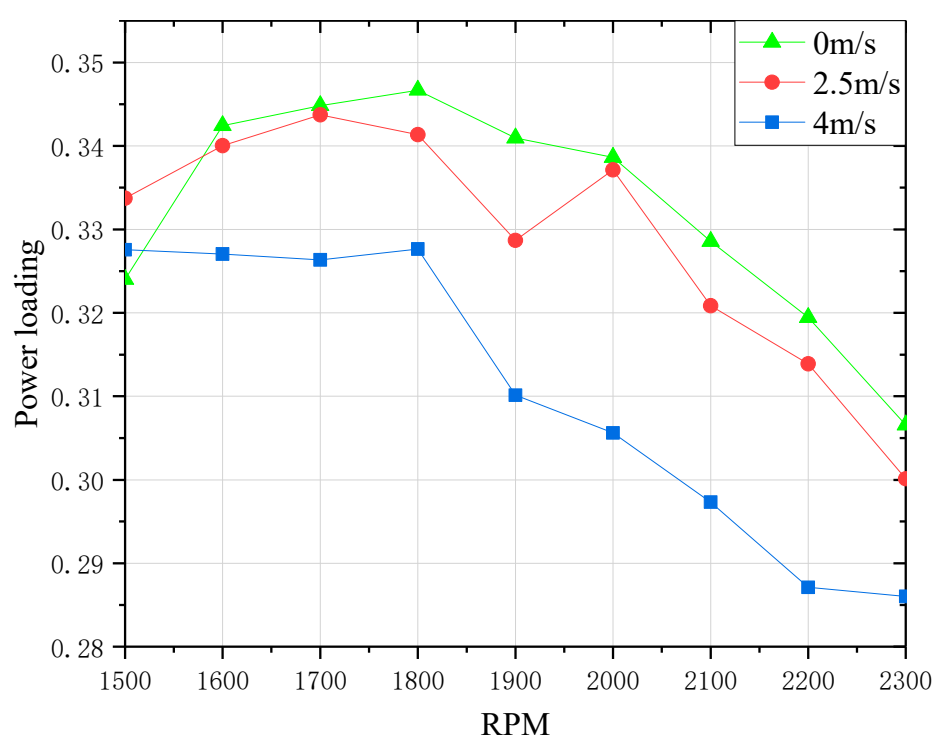

Figure 6. Power loading at different wind speeds.

According to Figure 6, PL decreased when the horizontal airflow was introduced, and the PL at $4 \mathrm{~m} / \mathrm{s}$ achieved minimum. Also, as the speed increased, the Hex-rotor UAV achieved the maximum power loading value at 1800 RPM. In this case, the increase of the horizontal airflow enhanced the aerodynamic interference of the Hex-rotor UAV, which led to the decrement of the power loading. Therefore, it can be inferred that the smaller airflow and low speed ( $2.5 \mathrm{~m} / \mathrm{s}$ and $4 \mathrm{~m} / \mathrm{s}, 1500 \mathrm{RPM})$ are beneficial to improve the aerodynamic characteristics of the Hex-rotor UAV.

During the experiments, the rotation speed and thrust of the Hex-rotor UAV were measured by the optical tachometer and the thrust sensor, respectively. According to the Kline-McClintock equations, uncertainty of the thrust coefficient is [21-23]:

$$
\Delta C_{T}=\sqrt{\left(\frac{C_{T}}{T} \cdot \Delta T\right)^{2}+\left(\frac{-2 C_{T}}{\Omega} \cdot \Delta \Omega\right)^{2}}
$$

Therefore,

$$
\frac{\Delta C_{T}}{C_{T}}=\sqrt{\left(\frac{\Delta T}{T}\right)^{2}+4\left(\frac{\Delta \Omega}{\Omega}\right)^{2}}
$$

Similarly, the uncertainty of the power coefficient $C_{P}$ and power loading $P L$ is expressed as:

$$
\begin{gathered}
\frac{\Delta C_{P}}{C_{P}}=\sqrt{\left(\frac{\Delta Q}{Q}\right)^{2}+4\left(\frac{\Delta \Omega}{\Omega}\right)^{2}} \\
\frac{\Delta P L}{P L}=\sqrt{\left(\frac{\Delta T}{T}\right)^{2}+\left(\frac{\Delta Q}{Q}\right)^{2}+\left(\frac{\Delta \Omega}{\Omega}\right)^{2}}
\end{gathered}
$$

Therefore, the uncertainties in $C_{T}, C_{P}$, and $P L$ are $1.2 \%, 1.1 \%$ and $1.5 \%$, respectively.

\section{Computational Fluid Dynamics (CFD) Simulation}

\subsection{Simulation Setup}

Computational fluid dynamics (CFD) are a very effective way to present the mutual interference of structures intuitively. Considering the highly unstable airflow around the Hex-rotor UAV, the sliding grid is applied in the simulation. The mesh distribution is shown in Figure 7. 


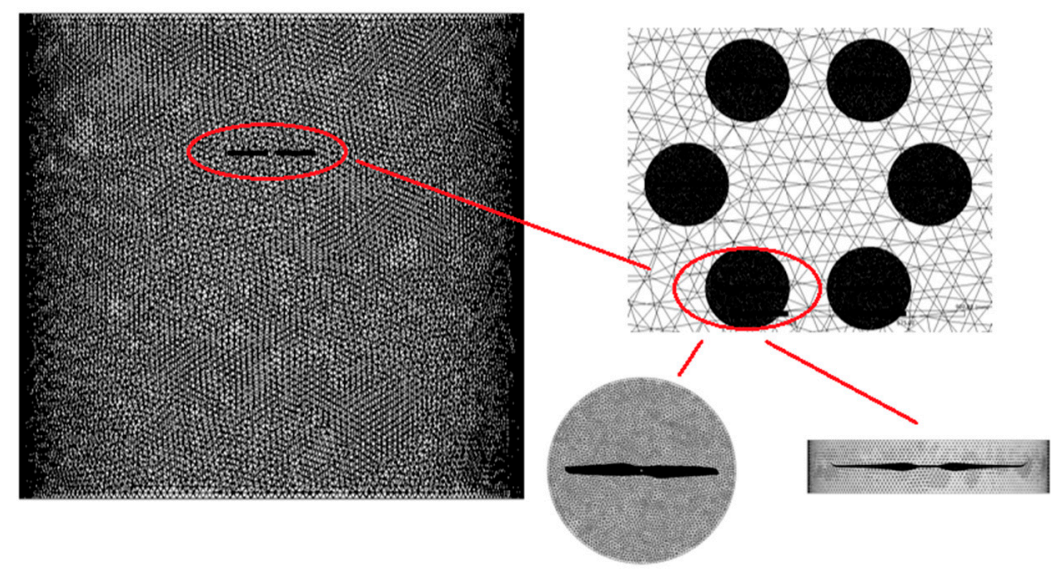

Figure 7. Mesh distribution.

\subsection{Streamline Distribution}

Streamline distribution of the flow field is shown in Figure 8.

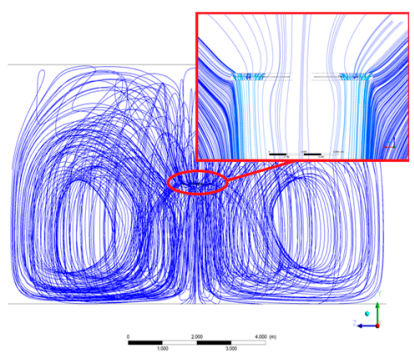

(a)

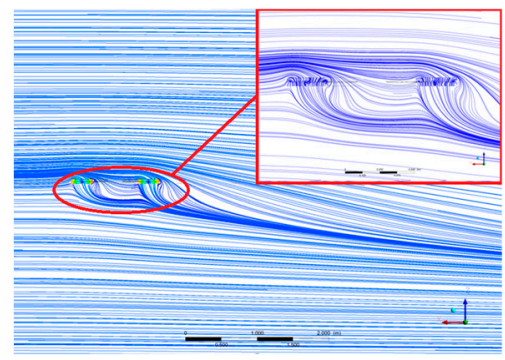

(b)

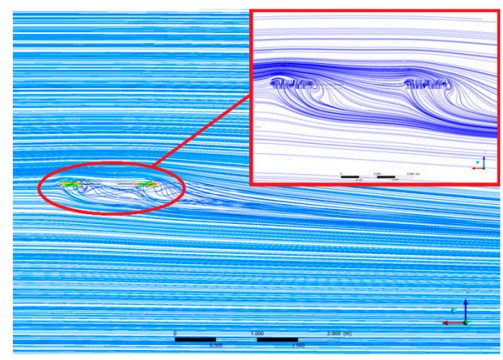

(c)

Figure 8. Streamline distribution (a) $0 \mathrm{~m} / \mathrm{s}$ (b) $2.5 \mathrm{~m} / \mathrm{s}$ (c) $4 \mathrm{~m} / \mathrm{s}$.

It is clear that the streamlines were diffused by the vortices, causing mutual interference between the rotors when the horizontal airflow was introduced in the fluid field. As the wind speed increased, the interference between the vortices became stronger, the streamlines began to couple with each other, and eventually it may cause vibration when the tip vortex is completely diffused. Combined with the experiment results, it can be inferred that the power loading will be greatly affected by the horizontal airflow with a higher power consumption, which may reduce the aerodynamic performance of the Hex-rotor UAV.

\subsection{Pressure Distribution}

The pressure contour on the plane upper $10 \mathrm{~mm}$ of the center of the Hex-rotor UAV is shown in Figure 9. The pressure contour on the center of the Hex-rotor UAV is shown in Figure 10. The pressure distribution on the plane lower $10 \mathrm{~mm}$ of the center of the Hex-rotor UAV is shown in Figure 11. 


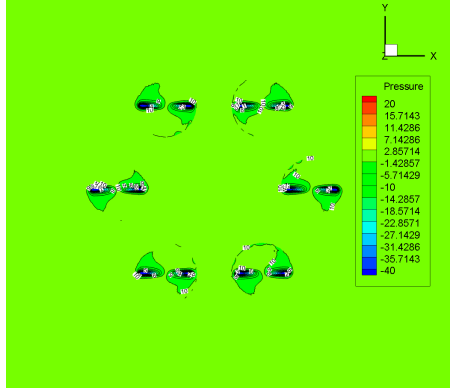

(a)

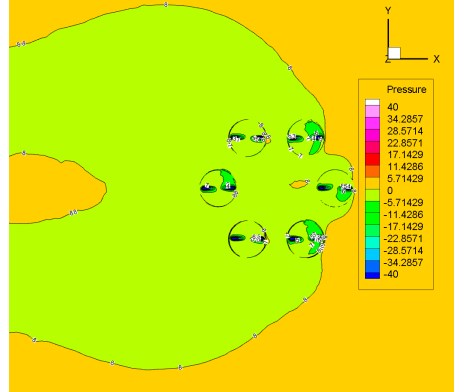

(b)

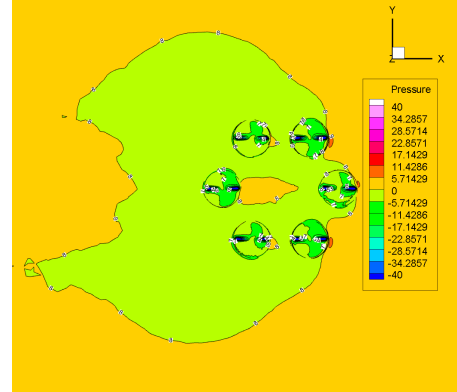

(c)

Figure 9. Pressure distribution on the plane upper $10 \mathrm{~mm}$ of the center of the Hex-rotor unmanned aerial vehicle (UAV) (a) $0 \mathrm{~m} / \mathrm{s}$ (b) $2.5 \mathrm{~m} / \mathrm{s}$ (c) $4 \mathrm{~m} / \mathrm{s}$.

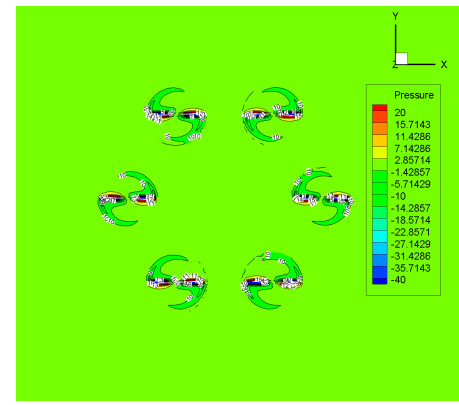

(a)

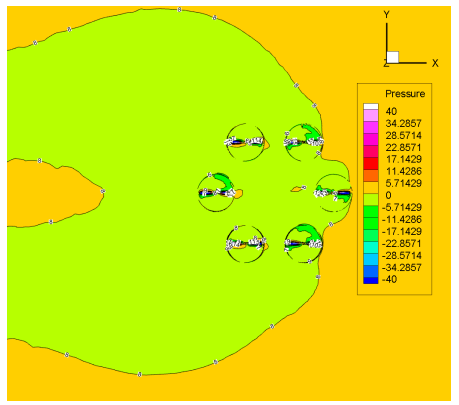

(b)

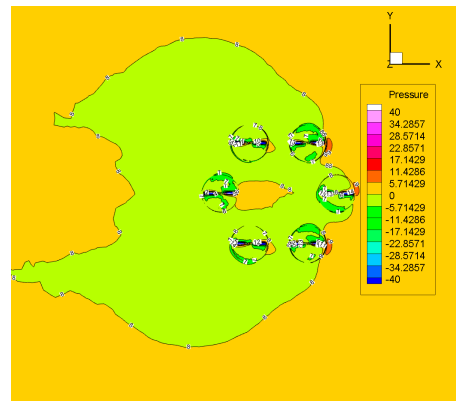

(c)

Figure 10. Pressure distribution on the center plane of the Hex-rotor UAV (a) 0 m/s (b) 2.5 m/s (c) 4 m/s.

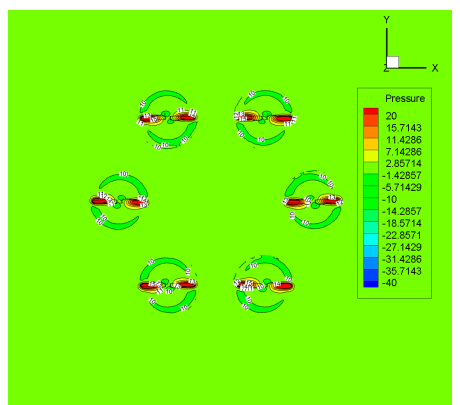

(a)

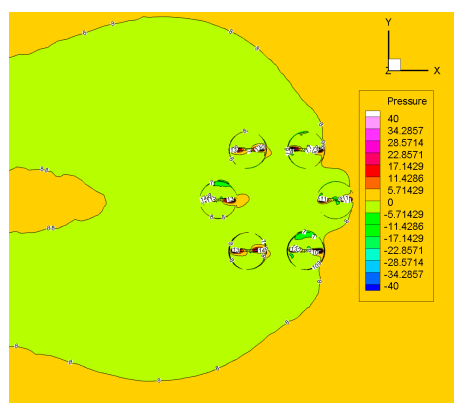

(b)

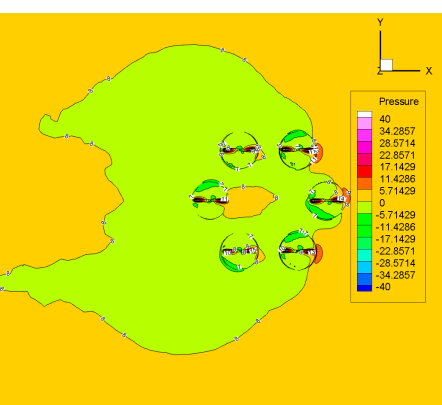

(c)

Figure 11. Pressure distribution on the plane upper $10 \mathrm{~mm}$ of the center of the Hex-rotor UAV (a) $0 \mathrm{~m} / \mathrm{s}$ (b) $2.5 \mathrm{~m} / \mathrm{s}$ (c) $4 \mathrm{~m} / \mathrm{s}$.

In Figure 9, the Hex-rotor UAV with different horizontal airflows had different negative pressure regions above the rotor. With the increase in the horizontal airflow, the pressure above the rotor was chaotic, and a positive pressure region appeared in the horizontal airflow direction.

As shown in Figure 10, the pressure distribution between the rotors was uniform at $0 \mathrm{~m} / \mathrm{s}$. The pressure change around the rotor was not obvious when the horizontal airflows were involved, but the pressure change around the rotor achieved the maximum, especially at the rotor tip.

According to Figure 11, the positive pressure region under the Hex-rotor UAV had a tendency to gradually decrease with the gradual increase of the horizontal airflow.

Combined with the experiment results, it can be inferred that uniform pressure distribution is a necessary condition for the Hex-rotor UAV to have a higher hovering efficiency (power loading). 


\subsection{Velocity Distribution}

The velocity contour between the rotors with the influence of horizontal airflow is shown in Figure 12.

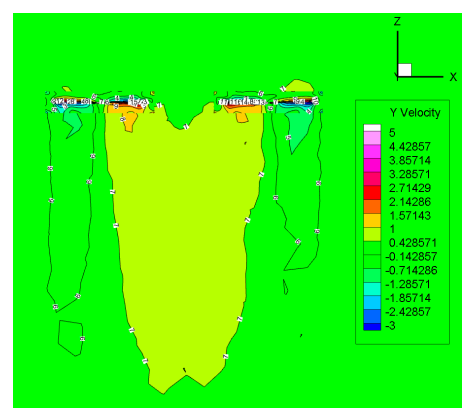

(a)

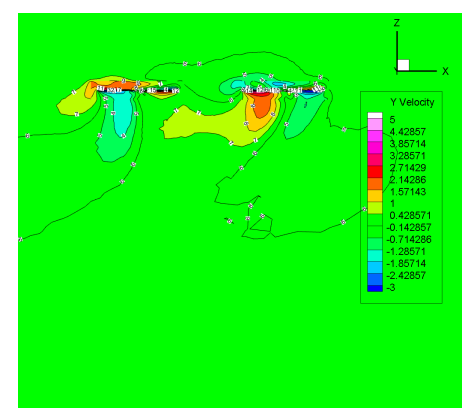

(b)

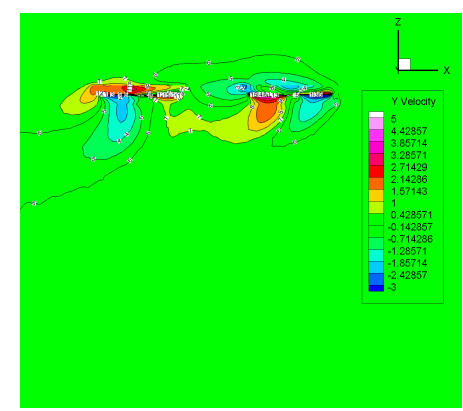

(c)

Figure 12. Velocity contour distribution (a) $0 \mathrm{~m} / \mathrm{s}$ (b) $2.5 \mathrm{~m} / \mathrm{s} \mathrm{(c)} 4 \mathrm{~m} / \mathrm{s}$.

It is clear that the vortices easily shed from the tip of the rotor due to the coupled interference between the rotors. As shown in Figure 12a with the horizontal airflow at $0 \mathrm{~m} / \mathrm{s}$, the vortices that shed are below the Hex-rotor UAV. As shown in Figure 12b,c, as the horizontal airflow increased, the vortices position of the detachment moved along the wind direction. Therefore, the vortices shedding of the tip of the blade was affected by the airflow in the horizontal direction relative to the no-horizontal airflow, which reduced the aerodynamic performance of the Hex-rotor UAV.

\subsection{Vortex Distribution}

The vortex distribution of the Hex-rotor UAV is shown in Figure 13.

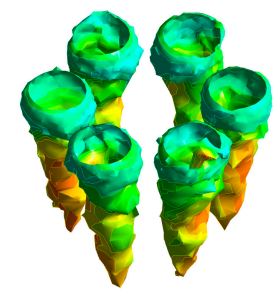

(a)

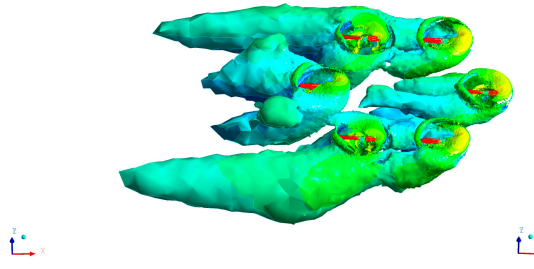

(b)

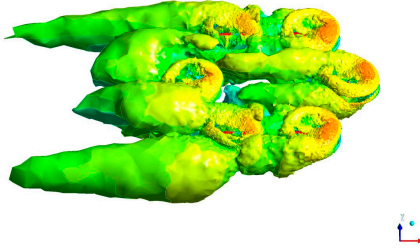

(c)

Figure 13. Vortex distribution of Hex-rotor UAV (a) 0 m/s (b) 2.5 m/s (c) 4 m/s.

When the horizontal airflow increased, the interference between the rotors of the Hex-rotor UAV was significantly enhanced and the velocity changes were more obvious. In the vortex diagram with a horizontal airflow of $2.5 \mathrm{~m} / \mathrm{s}$ and $4 \mathrm{~m} / \mathrm{s}$, the left airflow changed the vortex direction of the left rotor which affected the vortex change on the right side, and this interference gradually increased with the increase of horizontal flow.

Based on the simulation analysis and experimental results, it can be inferred that the movement and deformation of the deteriorated vortices can change the aerodynamic performance of the Hex-rotor UAV in the horizontal airflow.

\section{Conclusions}

This paper presented the aerodynamic performance of a Hex-rotor UAV considering the horizontal airflow by experiments and numerical simulations. Combined with the results of low-speed wind tunnel experiments, the aerodynamic performance of a Hex-rotor UAV with different horizontal airflow $(0 \mathrm{~m} / \mathrm{s}, 2.5 \mathrm{~m} / \mathrm{s}, 4 \mathrm{~m} / \mathrm{s})$ is analyzed in detail. Conclusions are as follows: 
1. The pressure and velocity distribution proved that the deformation of the downwash resulting from the horizontal airflow will increase the power consumption, indicating that the aerodynamic performance of the Hex-rotor UAV is determined by the instant thrust variation. This is because when horizontal airflow is introduced, the streamlines are squeezed and coupled with each other and the maximum pressure region of the rotor may have the maximum thrust and a higher power loading.

2. For a lower rotor speed (1500-1900 RPM), the Hex-rotor UAV showed a good hover efficiency characterized by a higher power loading, especially at 1700-1800 RPM. In fact, the horizontal airflow increased mutual coupling of the vortices and improved the aerodynamic performance by maintaining a higher thrust. This can be useful in the aerodynamic design of a Hex-rotor UAV for wind resistance.

3. For a higher rotor speed (1900-2300 RPM), the hover efficiency decreased with a thrust decrement and power increment as compared to the case at $0 \mathrm{~m} / \mathrm{s}$. Actually, the stronger coupling interference began to move along with the wind direction, which caused the rotor vibration, especially around the rotor tip. In this case, reactions will need to include increasing power, increasing angle of attack, sideslip, etc. in a quasi-steady manner to maintain the desired flight path when the rotor speed is higher than 1900 RPM, especially for the work mode at 2200 RPM.

4. The aerodynamic performance of the Hex-rotor UAV is also related to the vortices movement or deformation of the blade tip. The streamline and vortices distribution at $2.5 \mathrm{~m} / \mathrm{s}$ showed that the proper airflow may improve the aerodynamic performance by increasing the thrust, which also indicated that the Hex-rotor UAV will be simply manipulated by changing the rotor speed in a light breeze. On the other hand, the controller manipulation will be introduced in the gentle breeze with a higher wind speed. In addition, further research will involve a higher wind speed to study the wind resistance of the Hex-rotor UAV.

Author Contributions: Y.L. carried out experiments and analyzed experimental results; M.C. wrote the manuscript with assistance of Y.L.

Funding: This research was supported by the National Natural Science Foundation of China, grant No. 51505087, the State Scholarship Fund from China Scholarship Council, grant number 201806655008, and Fujian Provincial Industrial Robot Basic Components Technology Research and Development Center, grant number 2014H21010011.

Acknowledgments: The authors thank the Key Laboratory of Fluid Power and Intelligent Electro-Hydraulic Control (Fuzhou University), Fujian Province University, and Fuzhou University Jinjiang Science and Education Park for applying the experimental field.

Conflicts of Interest: The authors declare no conflicts of interest.

\section{References}

1. Mai, Y.; Zhao, H.; Guo, S. The Analysis of Image Stabilization Technology Based on Small-UAV Airborne Video. In Proceedings of the 2012 International Conference on Computer Science and Electronics Engineering, Hangzhou, China, 23-25 March 2012.

2. Hoffmann, G.; Huang, H.; Waslander, S.; Tomlin, C. Quadrotor Helicopter Flight Dynamics and Control: Theory and Experiment. In Proceedings of the AIAA Guidance, Navigation, \& Control Conference and Exhibit, Hilton Head, SC, USA, 20-23 August 2007.

3. Pounds, P.; Mahony, R.; Corke, P. Modelling and control of a large quadrotor robot. Control Eng. Pract. 2010, 18, 691-699. [CrossRef]

4. Salazar, S.; Romero, H.; Lozano, R.; Castillo, P. Modeling and Real-Time Stabilization of an Aircraft Having Eight Rotors. J. Intell. Robot. Syst. 2009, 54, 455-470. [CrossRef]

5. Arellano-Muro, C.A.; Luque-Vega, L.F.; Castillo-Toledo, B.; Loukianov, A.G. Backstepping control with sliding mode estimation for a hexacopter. In Proceedings of the 2013 10th International Conference on Electrical Engineering, Computing Science and Automatic Control (CCE), Mexico City, Mexico, 30 September- 4 October 2013. 
6. Chen, X.; Wang, L. Cascaded model predictive control of a quadrotor UAV. In Proceedings of the Control Conference, Fremantle, WA, Australia, 4-5 November 2013.

7. Pflimlin, J.M.; Soueres, P.; Hamel, T. Position control of a ducted fan vtol uav in crosswind. Int. J. Control 2007, 80, 666-683. [CrossRef]

8. Ma, Q.D.; Sun, Z.G.; Wu, J.R.; Zhang, W.Z. Dynamic modeling for a miniature six-rotor unmanned aerial vehicle. Appl. Mech. Mater. 2013, 321-324, 819-823. [CrossRef]

9. Lee, J.Y.S.; Leang, K.K.; Yim, W. Design and Control of a Fully-Actuated Hexrotor for Aerial Manipulation Applications. J. Mech. Robot. 2018, 10. [CrossRef]

10. Zhang, Z.; Liu, Z.; Wen, N. Research on Adaptive Backstepping Sliding Mode Control Method for a Hex-rotor Unmanned Aerial Vehicle. In Proceedings of the 2016 IEEE Chinese Guidance, Navigation and Control Conference (IEEE CGNCC2016), Nanjing, China, 12-14 August 2016.

11. Shi, T.; Wang, H.; Cui, W.; Ren, L. Indoor path planning for hex-rotor aircraft with landmark-based visual navigation. In Proceedings of the 12th International Conference on Natural Computation, Fuzzy Systems and Knowledge Discovery (ICNC-FSKD), Changsha, China, 13-15 August 2016.

12. Kirsch, B.; Alexopoulos, A.; Badreddin, E. Non-linear model based control and parameter identification of a hex-rotor UAV. In Proceedings of the IEEE International Conference on Systems, Man, and Cybernetics (SMC2016), Budapest, Hungary, 9-12 October 2016.

13. Zhao, C.J.; Bai, Y.; Gong, X.; Xu, D.F.; Xu, Z.J. Control System Design of a Hex-Rotor Aircraft Based on the Neural Network Sliding Mode Method. Adv. Mater. Res. 2014, 971-973, 418-421. [CrossRef]

14. Tan, Y.; Chen, J.; Norton, T.; Wang, J.; Liu, X.; Zheng, Y.; Yang, S. The computational fluid dynamic modeling of downwash flow field for a six-rotor uav. Front. Agric. Sci. Eng. 2018, 5, 159-167.

15. Hrishikeshavan, V. Experimental Investigation of a Shrouded Rotor Micro Air Vehicle in Hover and in Edgewise Gusts. Doctoral Dissertation, University of Maryland, College Park, MD, USA, 2011. Available online: http://hdl.handle.net/1903/12351 (accessed on 17 February 2012).

16. Chang-Jun, Z.; Yue, B.; Hun, G.; Cheng, P. Hex-rotor unmanned aerial vehicle controller and its flight experiment under aerodynamic disturbance. Opt. Precis. Eng. 2015, 23, 1088-1095. [CrossRef]

17. Lei, Y.; Wang, J.L. Aerodynamic Performance of Quadrotor UAV with Non-Planar Rotors. Appl. Sci. 2019, 9, 2779. [CrossRef]

18. Lei, Y.; Lin, R. Effect of wind disturbance on the aerodynamic performance of coaxial rotors during hovering. Meas. Control 2019, 52, 665-674. [CrossRef]

19. Langford, J.S. The Daedalus Project: Summary of Lessons Learne. In Proceedings of the Aircraft Design and Operations Meeting, Seattle, WA, USA, 31 July-2 August 1989.

20. Bohorquez, F.; Samuel, P.; Sirohi, J.; Pines, D.; Rudd, L.; Perel, R. Design, Analysis and Hover Performance of a Rotary Wing Micro Air Vehicle. J. Am. Helicopter Soc. 2003, 48, 80-90. [CrossRef]

21. Gur, O.; Rosen, A. Optimizing electric propulsion systems for unmanned aerial vehicles. J. Aircr. 2009, 46, 1340-1353. [CrossRef]

22. Onda, M.; Nanba, M.; Matsuuchi, K.; Furui, J.; Sasaki, A. Stratospheric LTA Platform and its Thrust Efficiency with a Stern Propeller. In Proceedings of the 1st UAV Conference, Portsmouth, VA, USA, 20-23 May 2002.

23. Slutsky, B.A. Compensation of an elastic error in force measurement. AIAA J. 2005, 43, 1326-1335. [CrossRef]

(C) 2019 by the authors. Licensee MDPI, Basel, Switzerland. This article is an open access article distributed under the terms and conditions of the Creative Commons Attribution (CC BY) license (http://creativecommons.org/licenses/by/4.0/). 